Meta

Journal des tradlucteurs

Translators' Journal

\title{
Essai de terminologie politique contrastive (français-anglais) sur les thèmes de « liberté " et de « volonté "
}

\section{Paul Jinot}

Volume 35, numéro 2, juin 1990

URI : https://id.erudit.org/iderudit/003266ar

DOI : https://doi.org/10.7202/003266ar

Aller au sommaire du numéro

\section{Éditeur(s)}

Les Presses de l'Université de Montréal

\section{ISSN}

0026-0452 (imprimé)

1492-1421 (numérique)

Découvrir la revue

\section{Citer cet article}

Jinot, P. (1990). Essai de terminologie politique contrastive (français-anglais) sur les thèmes de « liberté » et de " volonté ». Meta, 35(2), 398-406. https://doi.org/10.7202/003266ar d'utilisation que vous pouvez consulter en ligne. 


\title{
ÉTUDES TERMINOLOGIQUES ET LINGUISTIQUES
}

\begin{abstract}
ESSAI DE TERMINOLOGIE POLITIQUE CONTRASTIVE (FRANÇAISANGLAIS) SUR LES THĖMES DE «LIBERTÉ» ET DE «VOLONTÉ»
\end{abstract}

1.1 Nous savons que les unités lexicales, loin d'être sémantiquement simples, sont en fait des faisceaux de traits sémantiques. C'est ce qui fait que, dans une langue donnée, les vrais synonymes sont rares, et, d'une langue à l'autre, les équivalences lexicales parfaites pratiquement inexistantes. C'est la présence ou l'absence de tel trait sémantique qui introduit la nuance sémantique ou stylistique, plus ou moins marquée, qui permet de distinguer deux termes analogues dans une langue donnée. De même, le clivage qui caractérise tant de «faux-amis» de l'anglais et du français tient au fait que, à partir de cette «molécule» unique que représentait le terme emprunté ${ }^{1}$, chaque langue a privilégié des «atomes» différents, qui ont fini par polariser les termes en question. Le bicentenaire récent de la Constitution des États-Unis, celui, tout proche, de la Révolution française, et les élans de grandiloquence que de telles célébrations ne manquent jamais d'inspirer, fournissent un excellent prétexte pour examiner, dans une perspective comparative, les avatars qu'ont subis, dans les deux nations en question, certains des termes clés du jargon idéologique qu'elles paraissent avoir en commun. Dans le cadre du présent article, je m'en tiendrai à l'analyse du couple volontarisme (français) - voluntarism (anglais), analyse en vue de laquelle je serai amené à enfoncer quelques portes ouvertes, en particulier à propos d'un autre couple, beaucoup plus visible et mieux connu, liberal (anglais)/libéral (français).

1.2 Sans céder à la tentation du solipsisme, force est de reconnaitre que chaque locuteur d'une langue a sa propre interprétation, qui repose sur une expérience personnelle unique, des unités lexicales dont il se sert. Dans la pratique, sur le plan du concret, l'univers du réel impose un système de références objectives communes (designata) qui permet aux éléments idiosyncratiques de se résorber de telle sorte que la communication puisse avoir lieu sans trop de flou et de bavures. Les termes abstraits, qui ne bénéficient pas, eux, de cette possibilité de recentrage, et qui sont en outre fréquemment chargés de connotations émotives, sont beaucoup plus susceptibles de dérive sémantique anarchique ${ }^{2}$.

1.3 C'est en particulier le cas des termes à contenu idéologique, dont le climat culturel et en particulier politique dans lequel ils évoluent tend par ailleurs à monter en épingle les sèmes à aspect affectif, connotatif, au détriment des sèmes à aspect cognitif, intellectuel. C'est ce qui en fait d'excellents outils de la propagande et de son réductionnisme manichéen ou de sa langue de bois. À l'examen, nos clichés les plus sacro-saints révèlent un potentiel orwellien d'une extraordinaire richesse; nous constatons que démocratie a deux sens antagonistes, selon qu'elle est «populaire» ou «occidentale»; que liberté, «historiquement, (...) signifie pour les uns romantisme et contestation de l'ordre établi, alors que pour les autres elle est l'ordre, fondement voulu et vénéré d'un système social dont la légitimité n'est que marginalement mise en doute ${ }^{3} »$, qu' «il faut bien distinguer la liberté naturelle qui n'a pour bornes que les forces de l'individu, de la liberté civile qui est limitée par la volonté générale ${ }^{4}$, et que, de toute facon, «quiconque refusera d'obéir à la volonté générale y sera contraint par tout le corps; ce qui ne signifie autre chose sinon qu'on le forcera à être libre ${ }^{5}$; que l'égalité est relative, comme l'a noté l'auteur de 
Animal Farm («all animals are equal, but some animals are more equal than others», p. 114) et de 1984, ouvrage qui illustre de façon plus frappante encore cette appropriation du langage (Newspeak) par une oligarchie qui fait profession de cultiver le paradoxe et l'oxymoron:

\author{
(...) the three slogans of the Party: \\ WAR IS PEACE \\ FREEDOM IS SLAVERY \\ IGNORANCE IS STRENGTH (p. 17);
}

2.1 Lorsque le dérapage se produit dans des directions différentes, parfois radicalement opposées, ces termes homomorphes constituent de redoutables chausse-trappes pour le lecteur et le traducteur allophone. Un des cas typiques est sans doute celui de l'adjectif anglais liberal (et ses dérivés, liberalism, liberalization, ...) avec son homomorphe français libéral (etc.). Le lien qui les relie à leur racine latine liber, à la notion de «liberté», se tend et se relâche selon le type de libertés auxquelles ils se rapportent, qui ne sont pas nécessairement les mêmes dans les deux langues:

On social issues (...), American attitudes betray no evidence of a right turn. In general, religion, feminism, civil liberties, abortion, and race relations are the policy areas in which the public has shown the sharpest increase in liberalism since the Second World War ${ }^{6}$.

Dans ce contexte «social» (excluant les considérations économiques), celui des libertés civiles, liberalism est pratiquement synonyme de tolerance/tolérance (Liberal A. Adj. ...4. «free from narrow prejudice, open-minded», S.O.E.D.) et équivaut à libéralisme, au sens $\left(3^{\circ}\right)$ du Petit Robert: "Attitude de respect à l'égard de l'indépendance d'autrui, de tolérance envers ses opinions V. Tolérance». Mais ce parallélisme ne se prolonge pas jusqu'aux domaines de l'économie et de la politique.

2.2 Libéralisme, que le Petit Robert qualifie de «Vieilli» au sens $\left(1^{\circ}\right)$ de «attitude, doctrine des libéraux, partisans de la liberté politique, de la liberté de conscience», s'emploie effectivement surtout de nos jours au sens $\left(2^{\circ}\right)$ du Petit Robert: «(Opposé à étatisme, socialisme). Doctrine selon laquelle la liberté économique, le libre jeu de l'entreprise ne doivent pas être entravés». L'opposition «libéralisme-socialisme» n'est pas simplement académique; elle est tout à fait en évidence dans la politique française contemporaine, où les velléités d'《ouverture» du gouvernement Mitterrand-Rocard actuel inspirent cette réaction de Michel Prigent:

«(...) Si la droite et la gauche disent la même chose sur tout, s'il suffit au libéralisme d'être social pour être crédible ou au socialisme de reconnaître le marché pour être acceptable, la démocratie, loin d'y gagner, est sans doute menacée... Une démocratie est forte quand elle organise la confrontation des idées... Le risque actuel est d'allier un minimum d'efficacité libérale et un minimum de justice socialiste pour obtenir un maximun d'immobilisme... Le socialisme connut en 1968 une «majorité idéologique» et en 1981 une «majorité sociologique». Il gouverne aujourd'hui avec une «majorité relative». Si les conservateurs et les libéraux ne font pas attention, ils connaîtront le même mouvement... dans la «minorité», ce qui est moins agréable ${ }^{7}$,

réaction qui fait écho à celle d'un des représentants les plus éminents de cette «minorité», ancien président de la République :

«Intime conviction ou choix de stratège, M. Valéry Giscard d'Estaing a manifestement tiré un trait sur l'ouverture... L'ancien président abandonne l'objectif des «deux Français sur trois» pour revenir à une lecture bipolaire de la vie politique française de plus en plus inspirée d'ailleurs du modèle américain, la théorie des deux grands ensembles: social-démocrate 
à gauche, libéral et centriste à droite, pour préparer, selon ses vœux, «l'alternance dans une opposition constructive». (Le Monde, 1/9/88, p. 7)

À la limite, le libéralisme devient «libertaire/libertarian», sur le plan des libertés civiques et politiques (anarchie), ou revêt la forme du «capitalisme sauvage» sur le plan économique. Certaines de ses manifestations contemporaines les plus en vue s'appellent, du nom de leur promoteur, Thatcherisme et Reaganisme/Reaganomie (angl. «Reaganomics») :

«Le néo-libéralisme reaganien... En 1980, M. Reagan l'emporte grâce... à un programme négatif qui s'inscrit contre les déformations du libéralisme des précédentes années [la présidence du démocrate Carter]. Ses propositions - réductions d'impôts, déréglementation, diminution des dépenses sociales et augmentation des dépenses militaires - $(. . .)^{8}$ »

L'association étymologique de libéralisme avec «libre/liberté» persiste ainsi dans le contexte de la politique économique, où elle se traduit par le désengagement de l'État sur le plan économique et social, le recul de l'État-providence («réductions d'impôts, déréglementation - angl. deregulation -, diminution des dépenses sociales - angl. social welfare programs, social-spending programs»), d'une manière générale, le «moins d'État» («less government»). Le terme français correspond d'ailleurs le plus souvent à une locution anglaise contenant l'adjectif free, la version anglo-saxonne de libre: free market (economy), free enterprise, free trade, ... de même que des expressions comme libération (des échanges, par exemple) se traduisent le plus souvent par ce même adjectif à la forme comparative: free trade,... autant d'expressions anglaises qui caractérisent en fait paradoxalement la doctrine des «conservatives» beaucoup plus que celles des «liberals».

2.3 Ce libéralisme «Opposé à étatisme, socialisme» et quasi-synonyme de «conservateur» s'oppose aussi à son homomorphe anglais, tel que le S.O.E.D. le définit («Liberal A. adj....5. Of political opinions : Favourable to changes and reforms tending in the direction of democracy. Hence, epithet of a party; opp. to Conservative...»), ce qu'illustre fort bien la citation suivante, qui définit à sa manière ces notions de «conservative» et «liberal», dans le contexte américain (c'est moi qui souligne):

«American public opinion has long been described as both ideologically conservative and programmatically liberal. That is, Americans are opposed to big government, and respond favorably to the myths and symbols of competitive capitalism in the abstract. When it comes to assessing specific government programs or the behavior of actual business enterprises, however, they support government spending in a variety of domestic areas and are profoundly suspicious of big business. Similarly, Americans are strongly anti-communist... This basic opinion structure reflects voter adaptation to a political system in which the government is interventionist but nonsocialist ${ }^{9} . »$

Si l'on convient que les «réductions d'impôts» font aisément l'unanimité, en marge de toute idéologie, le seul élément commun de ce «liberalism» avec «le néo-libéralisme reaganien» mentionné plus haut porterait, à la rigueur, sur le niveau des dépenses militaires dans le contexte de la guerre froide. Partout ailleurs, ils se heurtent de front: «déréglementation» s'oppose à «they are suspicious of big business... public skepticism toward business, and support for government regulation of it...» (Ibid.) tout comme «diminution des dépenses sociales» à «they support government spending in a variety of domestic areas».

2.4 En fait, l'ambivalence de l'opinion publique américaine décrite ci-dessus se retrouve chez les jeunes Français, objets du dernier sondage de l' Express $^{10}$, qui confondent allègrement «liberté» (libéralisme) et «égalité» — ou du moins, intervention gouvernementale 
visant à réduire les inégalités naturelles, à promouvoir plus de justice sociale, (l'anglais libéralism):

\section{Les valeurs}

Pour chacun des mots et des idées suivantes, dites s'il représente pour vous plutôt quelque chose que vous aimez, qui vous est sympathique, ou plutôt quelque chose que vous n'aimez pas?

\begin{tabular}{|c|c|}
\hline Sym & apathique \\
\hline otection sociale & $86 \%$ \\
\hline galité sociale & $82 \%$ \\
\hline Libéralisme & $67 \%$ \\
\hline ocialisme & $46 \%$ \\
\hline ationalisme & $41 \%$ \\
\hline
\end{tabular}

Résultats qui inspirent au rédacteur de l'article ce commentaire sarcastique :

«(..) les jeunes pèsent lourd... Et leurs valeurs prennent un sérieux coup de pompe. Une allure carrément conservatrice qui fiche un peu le bourdon. Supersympa, c'est tout ce qui relève, eh oui ! de l'État assistance: la protection et l'égalité sociale, le socialisme, la nationalisation... Adieu les anars! Et vive l'État Léviathan, mais à condition de baigner dans le libéralisme, précisent nos sondés fort paradoxaux». (p. 64)

Il n'y a là de «paradoxe» que si «libéralisme» signifie le contraire de liberalism.

Toute confusion entre ces deux termes, résultat d'une éventuelle traduction trop hâtive par exemple, aboutit au contresens pur et simple. Nous avons vu plus haut que, pour traduire libéral(isme), le français a souvent intérêt à faire appel à l'équivalent anglosaxon free. Sauf à être obligé à se livrer à la gymnastique que pratiquent les commentateurs francophones de la scène politique américaine, prisonniers de cette polarité ${ }^{11}$, il convient peut-être, pour éviter toute équivoque, de traduire le terme anglais au moyen de l'étiquette générique politique socio-économique de gauche, ou par ce que la terminologie politique française appelle radical/radicalisme ou, de plus en plus, social-démocrate/ social(e)-démocratie ${ }^{12}$ (voir citation du Monde du 1/9/88, Supra):

L'équation reaganienne est bien connue : davantage de marché = davantage d'efficacité.

L'équation social-démocrate aussi : davantage d'État-providence $=$ davantage d'égalité... ${ }^{13}$

Très loin de son étymologie, et sans aller jusqu'à s'identifier à socialism - tabou dans la terminologie gauchiste aux États-Unis pour des raisons psychologiques - liberalism recoupe les sens de reformism, welfare state, interventionism, et, en français, l'Étattutelle, l'Etat-providence, dirigisme, voire... volontarisme.

3.1 Le dictionnaire étymologique nous apprend qu'il existait en latin un adjectif voluntarius (dérivé du nom voluntas, -atis, devenu en français volonté), qui a donné naissance, en moyen français, à volontaire, puis à l'anglais voluntary, sur lesquels ont été respectivement formés, par dérivation savante relativement récente, les substantifs volontarisme et voluntarism. Loin d'être monolithiques, les termes qui jalonnent cette évolution révèlent à l'examen des fissures qui autorisent des interprétations divergentes. $D u$ Contrat social, déjà cité un peu plus haut, fait par exemple la distinction suivante touchant le concept de «volonté»: «(...) chaque individu peut comme homme avoir une volonté particulière contraire ou dissemblable à la volonté générale qu'il a comme citoyen» (p. 54). 
L'ambivalence se retrouve au niveau de l'adjectif français, dont le sémantisme s'oriente selon deux axes principaux :

a) la notion d'intention, d'effort, de discipline, de fermeté, de tension de l'être,... «qui résulte d'un acte de volonté (et non de l'automatisme, des réflexes ou des impulsions)» (Petit Robert)

mais aussi, paradoxalement, un peu en retrait,

b) celle de bon vouloir, de bonne volonté - «qui n'est pas l'effet d'une contrainte, qui n'est pas forcé» (Ibid), qui suggère au contraire la fantaisie, le libre arbitre, la liberté d'agir comme bon vous semble, à la limite, de «n'en faire qu'à sa tête. V. Capricieux, entêté» (Ibid).

3.2 Quant à voluntary, le S.O.E.D., s'il lui reconnaît le sens (a), le situe très bas, presque en marge, sur la liste des significations:

A. adj. I...4. Done of deliberate intent or purpose; designed, intentional 1495.

II..5. Of muscles, etc.: Acting in response to the volition; directing or controlling voluntary movements 1788

Pour toutes les autres acceptions, c'est le sens (b) qui est la dominante: je cite, en vrac: «without external constraint, ...unprompted, unforced, willing, ready,...». A. I. i. a et surtout b. vont même carrément à l'encontre du sens (a) de volontaire :

«a. Of feelings, etc.: Arising or developing in the mind without external constraint; purely

spontaneous. b. Of actions: Performed or done of one's own free will, impulse or choice...»

Faut-il désormais s'étonner que les substantifs anglais et français dérivés de ces adjectifs, volontarisme et voluntarism, de même étymologie et encore si proches par la forme tant graphique que phonologique, en soient arrivés à désigner des concepts antinomiques?

3.3 En anglais, le Webster's New Collegiate Dictionary cite comme deuxième sens l'acception psycho-philosophique ( $\ll \mathbf{2}$. a theory that conceives will to be the dominant factor in experience or in the world») qui repose, comme en français, sur la notion (a), car le terme «will» ne prête pas ici à équivoque. Sa première définition paraît ambiguë, dans la mesure où elle demeure prisonnière de la (légère) ambivalence de l'adjectif voluntary («1. the principle or system of doing something by or relying on voluntary action»); toutefois, cette ombre d'ambiguïté se dissipe totalement lorsqu'on note que le Webster propose cette acception de voluntarism («VOLUNTARISM 1») comme synonyme de «VOLUNTEERISM», lequel terme, tout comme sa racine volunteer, est dépourvu d'ambiguïté, mais au sens (b) cette fois. Si l'on ne retient que l'usage courant, on constate que, si volunteer ism est probablement plus fréquent (comme le suggère le numéro de juillet 1986 de Good Housekeeping («Twenty million readers every month», s'il vous plaît!), dont un des articles s'intitule justement «Volunteerism in America»), voluntarism doit être lui aussi bien vivace, à en juger par cet extrait d'une page publicitaire publiée dans le numéro de septembre 1985 de The Atlantic (c'est encore moi qui souligne):

\section{GIVING... CARING}

A proverb is a short sentence based on a long experience. There's an $18^{\text {th }}$ century proverb that says one volonteer is worth two who are pressed into service...

The volunteer spirit has always been a special feature of the American character... 
People volunteer in many ways and for many reasons...

In America, particularly, people are given great opportunities. Voluntarism has to do with giving something back. (p. 25)

3.4 En français, en revanche, c'est le sens (a) qui l'a emporté, dans des contextes d'ordre philosophique et psychologique (cf. le Petit Robert) d'abord, puis finalement, sociopolitique :

VOLONTARISME sm. (D'après les emplois en phil. en psychol.).

- Attitude de qqn (homme d'État, dirigeant, parti, etc.) qui proclame clairement et fermement ses intentions, qui mène une politique énergique, qui cherche à peser sur les événements plutôt qu'à se contenter de les subir ou de les observer ${ }^{14}$.

Cette polarisation se manifeste on ne peut plus clairement dans les citations suivantes (c'est moi qui souligne):

«Il s'agit de trouver la voie médiane entre le laisser-faire gros d'aucun avenir et le volontarisme destructeur...» (Le Monde, $7 / 4 / 83$, p. 2)

«Les actions correctrices à mener exigent donc un travail en profondeur. C'est le champ propre de la politique éducative, technologique et industrielle, de la planification, bref d'un volontarisme économique qui plaît aux «politiques», mais qu'ignore ou rejette le modèle libéral selon lequel la «main invisible» du marché est censée pourvoir seule à la prospérité économique à court terme comme à long terme.» (Ibid., 6/1/83, p. 28)

Elle tient peut-être à la nature du paysage politique français, comme en témoigne Jean-Jacques Servan-Schreiber, citant d'abord Jean Jaurès, une des grandes figures du socialisme français, qui résumait ainsi, dans son Introduction à l'histoire socialiste, ce que lui paraissaient être les buts du marxisme (c'est moi qui souligne):

«Marx a déclaré que, jusqu'ici, les sociétés humaines avaient été gouvernées par la fatalité, par l'aveugle mouvement des forces économiques... Les institutions, les idées n'ont pas été l'euvre consciente d'hommes libres, mais le reflet de la consciente vie sociale dans le cerveau humain... L'histoire humaine ne commencera véritablement que lorsque l'homme, échappant à la tyrannie des forces inconscientes, gouvernera par sa raison et sa volonté la production elle-même ${ }^{15}$ 》.

puis un «technocrate» moderne, M. Pierre Massé :

«Les ressorts de cette grande œuvre - le développement économique et social — sont la liberté et la volonté de l'homme. Ses instruments sont les plans, partiels ou globaux, privés ou publics, qui, sous les formes les plus diverses, ont pour contenu commun la conscience et l'intentionnalité, opposées aux fatalités et aux hasards... ${ }^{16 »}$

Le «défi» de J.-J. Servan-Schreiber, l'entreprise à laquelle il s'est attelé, c'est justement de tenter de concilier ces deux points de vue, de canaliser «la dialectique planmarché» (Ibid., p. 285) vers une synthèse fructueuse «à la française», car, ajoute-t-il (c'est toujours moi qui souligne, et trois des quatre termes et expressions soulignés constituent autant de variations sur le thème de volontarisme):

«(...) Il est permis de demander plus de planification et plus de liberté, sans tomber dans la logomachie électorale. (...) En dépit des habitudes de pensée de la droite et de la gauche, libération des initiatives et maîtrise des actes qui engagent l'avenir sont les deux branches d'une même émancipation... La logique du libéralisme et la logique de la planification sont également boîteuses parce que l'une et l'autre amputent les hommes de leur pouvoir créateur... 17 
3.5 Il est d'ailleurs remarquable de noter en passant combien cette polarisation des termes dans l'usage contemporain aux États-Unis et en France reflète la psyché respective de l'homo politicus américain et de son homologue français: «Les Français ne sont pas orientés comme les Américains vers le business... ils ont une mentalité plus collective qu'individuelle», lit-on dans un article intitulé «Comment redresser l'image que les Américains se font de l'industrie française ?» (Le Monde, 3/4/86). Aux États-Unis, dont l'idéologie traditionnelle est assise sur les concepts de «( rugged) individualism», «freedom/liberty», «self-reliance»,... la «volonté», par la voix de voluntary/voluntarily, demeure de l'ordre de l'initiative, du choix individuel. En France, cette même «volonté» devient tout à fait spontanément, et ce depuis Rousseau, en passant par Mirabeau, «la volonté générale / volonté du peuple», laquelle a toujours plus ou moins force coercitive, en face d'une «concurrence» moins souvent qualifiée de «libre» que du stigmatisant «sauvage». Voici, par exemple, ce qu'écrivait Jean-Pierre Chevènement en 1986, alors qu'il était ministre de l'Éducation nationale :

«La droite, qui n'avait pas de grande ambition pour l'Université avant 1981, propose aujourd'hui de faire basculer le système universitaire dans la concurrence sauvage... Si l'on veut que nos universités puissent accueillir deux millions d'étudiants au début du siècle prochain, on ne peut s'en remettre à la concurrence. Il faut une volonté qui soit celle du pays tout entier et, pour tout dire, une programmation. Et il y a des choix politiques à faire ${ }^{18}$ \%. ( $L e$ Monde, $1 / 3 / 86$, p. 1)

C'est un politicien socialiste, il est vrai, qui tient ces propos. Mais en fait le volontarisme est un invariant de l'histoire politique française et de ses aléas, sans doute accentué par une longue tradition de centralisation administrative, «a centralized bureaucracy that went back without a break almost to the early middle Ages and had been made by Jean-Baptiste Colbert (1619-1683) and Napoleon into a relatively smooth and well-oiled machine ${ }^{19}$ ». Même, beaucoup plus près de nous, le Général de Gaulle, qui n'avait pourtant rien d'un gauchiste..., mais lisons plutôt les exemples particulièrement appropriés que fournit le Dictionnaire des mots contemporains à l'article VOLONTARISTE (dont j'ai déjà cité la définition ci-dessus):

«Les volontarismes politiques interviennent pour corriger ces tendances «naturelles» du marché; dans un pays où les traditions centralisatrices et étatiques sont aussi fortes qu'en France, il est inévitable que les hommes au pouvoir soient les premiers à tenter d'effectuer cette correction (Martinet, 73). À la démarche «intellectuelle et libérale» de l'actuel chef de l'État, le président du R.p.r. oppose «le volontarisme» du Général de Gaulle» (E. 12/6/78).

4. Ainsi, en face de l'anglais voluntarism, qui met l'accent sur le libre choix de l'individu, et s'apparenterait plutôt au français libéralisme, se dresse le français volontarisme, qui, dans le contexte socio-politique où il est le plus usité, est sémantiquement aux antipodes du libéralisme. Cependant que, dans l'univers de la politique, où il s'oppose à conservative, l'anglais liberal (liberalism), se dissociant de son étymologie, tend justement, lui, vers le pôle volontariste. Toutes les citations, tant françaises qu'anglaises, utilisées ci-dessus illustrent cette polarisation d'une terminologie, dont l'analyse comparative fournit au traducteur harcelé par les faux-amis une mine d'équivalences extrêmement précieuse: dans ses occurrences (d'ailleurs relativement rares), l'anglais voluntarism, synonyme la plupart du temps de volunteerism, correspondra au français bénévolat plutôt qu'à volontarisme. Quant à ce dernier, souvent employé comme euphémisme pour planification/interventionnisme/dirigisme..., il pourrait alors se traduire par les équivalents anglais traditionnels de planning/interventionism et state/government control; toutefois, comme ces derniers termes ont une forte charge affective négative, j'aimerais proposer 
l'expression neutre, voire méliorative, de «purposive effort», telle qu'elle figure dans le passage suivant:

“(...) It was Europe's decision to break from the unreflecting lock step of nature and tradition that led to such audacious visions as that of equality and social justice, and to the conviction that poverty and drudgery can be eliminated by purposive effort ${ }^{20 "}$.

ou la variante «purposive social action», empruntée au titre d'un article, «The Unanticipated Consequences of Purposive Social Action», du sociologue américain Robert Merton, à propos duquel Benjamin DeMott écrit (c'est moi qui souligne) :

"(...) Two chapters of Social Theory and a famous paper titled "the Unanticipated Consequences of Purposive Social Action" dealt with the nature of the obstacles besetting planners and with the reasons for rejecting despair about planning... Among the factors handicaping attempts to predict and control the future was the tendency of predictions themselves to affect outcomes... Changes must be wrought patiently within the institutional fabric... ${ }^{21}$

La référence à «purpose» ainsi qu'à «planning» semble effectivement être un des éléments fondamentaux de toute description du «libéralism» américain dès avant FDR et le «New Deal» et la «War on Poverty» des années 60 (c'est moi qui souligne) :

Past experience suggested that the best way for federal government to increase opportunity for the poor was through major national efforts: lowering the unemployment rate, improving schools, undertaking public works, and eliminating discrimination. Sometimes the programs that tumed out to be the most effective at reducing poverty - like the Homestead Act, the Erie Canal, universal public education, and the GI Bill - weren't planned for that purpose, but nonetheless changed social and economic conditions in some important way ${ }^{22}$.

Selon ses tenants «social-démocrates», il repose sur des idéaux de «progress, humanitarianism, and justice,... societal responsability...(towards) the underclass,... a just society, (a) commitment to greater social liberty,... the will to self-sacrifice...», qui s'expriment en particulier sous forme du «social transfer (through welfare programs)», cependant que ses adversaires libéraux, mus par la morale de l'efficacité et la philosophie du «moins d'État», lui reprochent son «absurd «passion of compassion (as The Public Interest has characterized it) - soft, emotional, Rousseauistic» («Rediscovering complexity», op. cit., p. 73-74) ${ }^{23}$.

5. À sa manière, cette polarisation terminologique reflète le conflit entre ces deux principes contradictoires que sont en fin de compte, et en dépit des slogans démagogiques qui se plaisent à les associer, la liberté et l'égalité. Le discours politique semble particulièrement porté à la coloration affective, à l'ambiguïté, et à la manipulation sémantique. De par sa nature, et malgré les revendications de «parler vrai», il constitue le domaine privilégié du flou calculé, de l'équivoque, de l'euphémisme, de la langue de bois, du «parler creux». À un tel régime, la langue est mise à rude épreuve. C'est peut-être ce qui fait dire à Lewis Lapham: «the promise of human feeling on the part of any institution... debases the language and props up the effigy to whom George Orwell gave the name "Big Brother"» (Harper's, January 1989, p. 8). Espérons du moins qu'un citoyen - et un traducteur - averti saura en valoir deux ! ${ }^{24}$ 


\section{NOTES}

1. Le cas typique, qui concerne directement le sujet du présent article, étant celui de la multitude des termes français, la plupart abstraits, qui ont envahi le lexique anglais dans le sillage de Guillaume le Conquérant.

2. Les termes concrets n'échappent évidemment pas à l'évolution sémantique, mais celle-ci accompagne alors généralement une évolution culturelle ou technologique dans laquelle nous sommes tous simultanément et souvent inconsciemment partie prenante ; si nous prenons le temps de la réflexion et de l'analyse, nous pouvons retracer et justifier cette évolution, au nom d'une certaine logique des faits.

3. «Liberté, liberty... Chérie», Le Monde, $21 / 6 / 86$, p. 11, col. 1.

4. Du Contrat Social, J.-J. Rousseau, p. 55-56.

5. Ibid., p. 54.

6. "The Myth of America's Turn to the Right», The Atlantic, May 1986, p. 45.

7. «Démocratie de confrontation», Le Monde, $28 / 10 / 88$, p. 2. A noter ici que, contrairement à leurs équivalents anglais, ces «conservateurs et libéraux» mentionnés dans la dernière phrase de la citation ne se situent pas à deux extrémités opposées de l'éventail politique, mais qu'ils sont du même bord, qu'ils constituent, conjointement, l'opposition de droite.

8. «Les prémices d'une société de partenariat aux États-Unis», Le Monde diplomatique, novembre 1988, p. 4.

9. The Myth of America's Turn to the Right», op. cit., p.44.

10. $\mathrm{N}^{\circ} 1956,30$ décembre 1988, p. 70.

11. Par exemple, Bruno Dethomas, dans le Monde du 3 novembre 1988 : «La «reaganomie», puisque tel fut le nom donné à cette politique, était fondée sur le rejet des politiques keynésiennes... Les économistes libéraux (2) mettent pourtant en exergue les résultats de cette politique : repartie depuis 1982, l'économie américaine a enregistré depuis lors sa plus longue phase d'expansion depuis la guerre...» (p.7)

se fait un devoir de préciser, en notes:

(2) Au sens économique et non au sens politique selon lequel un «libéral» est, aux États-Unis, «de gauche»,

tout comme Jan Krauze, dans le même numéro du Monde, se sent obligé de remettre les pendules à l'heure, à l'intention du lecteur français:

«(...) l'Amérique de la fin des années Reagan reste convaincue que le conservatisme est une vertu et le libéralisme une tare - un libéral américain étant, en gros, un homme de gauche». (p. 6)

12. Selon le modèle des socialistes allemands, qui tentent d'«épurer la doctrine de ses fondements marxistes», selon J.-P. Pigasse (L'Express, 27 février 1987, p. 74), et concilier ainsi une politique de justice sociale et l'économie de marché - synthèse que J.-J. Servan-Schreiber appelait déjà de tous ses voux (cf. 3.4., Supra; voir aussi $L^{\prime}$ Express des 10 avril 1987, p. 9, et 17 avril 1987, p. 74; à comparer avec la citation de 2.3.).

13. La machine égalitaire, Alain Minc, p. 247.

14. Dictionnaire des mots contemporains, Col. Usuels du Robert, p. 732.

15. Le défi américain, p. 285-286.

16. Ibid., p. 286 .

17. libid., p. 285.

18. «L'école illustre en effet jusqu'au paroxysme le modèle démocratique français : égalitaire, unitaire, tutélaire, en totale contradiction avec les fondements individualistes, pluralistes des États-Unis ou de la GrandeBretagne» (Alain Minc, op. cit., p. 29.)

19. The Europeans, Luigi Barzini, Penguin Books (1984), p. 126, Et n'oublions pas le «Jacobinisme»!

20. «Nuclear Power's Faustian bargain», by Erazim Kohák, Harper's, May 1987, p. 16.

21. «Rediscovering Complexity», The Atlantic, September 1988, p. 70.

22. "The Unfinished War», The Atlantic, December 1988, p. 46.

23. Par exemple, tout récemment encore, «...during the campaign... Bush... mocked the generously idealistic tradition in American politics (a.k.a. the «L word»), and wondered why women who received abortions weren't being sent to prison» («Notebook», Lewis H. Lapham, Harper's, January 1989, p. 9). Mon propos étant non pas de prendre position dans un débat idéologique, mais simplement d'illustrer une des dimensions terminologiques d'un tel débat, je souhaite faire entendre ici un autre son de cloche, qui a d'ailleurs parfaitement sa place dans la perspective qui m'intéresse :

«À long terme, l'inégalité passe, d'une certaine façon, par la perpétuation du modèle social-démocrate, l'égalité par une étrange révolution qui ferait du marché un instrument d'une plus grande justice sociale». (Alain Minc, op. cit., p. 67)

24. Et, il va sans dire, une citoyenne - une traductrice — avertie deuses !

\section{BIBLIOGRAPHIE}

BARZINI Luigi (1984), The Europeans, Penguin Books.

MINC Alain (1987), La Machine égalitaire, Le livre de poche, Grasset Paris. ORWELL George (1976), Animal Farm, Penguin Books. 
ORWELL, George (1976), Animal Farm, Penguin Books.

ORWELL, George (1961), 1984, New American Library, New York.

Petit Robert (1986), Dictionnaires Robert, Paris et Montréal.

ROUSSEAU J.-J. (1966), Du Contrat Social, Garnier-Flammarion, Paris.

SERVAN-SCHREIBER J.-J. (1967), Le défi américain, Denoël, Paris.

The Shorter Oxford English Dictionary (1950), Clarendon Press, Oxford, England.

Webster's New Collegiate Dictionary (1981), Thomas Allen \& Son Limited, Toronto, Ontario. 